denses dans tout le fromag?, ce qui indique que les coques croissent, se reproduisent et attaquent le milieu environnant pour se nourrir.

Des 265 cultures isolées, 26 seulement furent identifiées comme des streptocoques différant du S. laetis. Le plus grand nombre de celles-ci provenaient des qualités inférieures de fromage, et une seule culture provenait de la meilleure qualité de fromage.

Il est probable que ce groupe est le moins abondant de tous dans le fromage de Cheddar. S'il joue un rôle important dans la maturation, c'est sans doute en attaquant la caséine en même temps que d'autres groupes, comme il a été indiqué dans les travaux d'Evans. celle-ci a trouvé que cerlains types de streptocoques, employés comme levains, avec d'autres types, avaient une influence appréciable sur le développement de l'aròme.

Le nombre de levures trouvées est si petit, 3 en tout, qu'on n'a pas examiné dans cet article leur ròle dans la qualité du fromage. Des 3 espèces, deux furent isolées des qualités inférieures de fromage, tandis qu'une seule espèce était trouvée dans la meilleure qualité de fromage. Après la terminaison du travail ci-dessus, on a cependant examiné un fromage de qualité inférieure acheté dans un marché, ét on l'a trouvé contenir une flore prédominante de levùrcs.

Dans la flore d'un fromage de Cheddar typique, le $S$. lactis se développe rapidement au débui, et, dans les meilleures qualités, les lactobacilles, coques et streptocoques, se développent dans les dernières phases de la maturation jusqu'au $4^{\mathrm{e}}$ ou $5^{\mathrm{e}}$ mois : à ce moment, le nombre total des bactéries devient relativement petit. Dans les qualités inférieures de fromage, les bâtonnets producteurs de spores et Gram-négatifs et, occasionnellement, les levures, se développent après la prédominance initiale du $S$. lactis.

\title{
LA QUESTION DU LAIT \\ DANS UN HÔPITAL-HOSPIGE D'UNE VILLE DE 20.000 ÂMES
}

\author{
par J. CASSEL,
}

Pharmacien en Chef de l'Hôpital de Dieppe (Seine-Inférieure).

A Dieppe, ville de 22.000 âmes, le service hospitalier est assuré par un seul établissement : " les hospices civils de Dieppe », à la fois hôpital et hospice. Le nombre des lits est de 600 . 
Les malades sont répartis en deux grandes catégories : $1^{\circ}$ Service de médecine, dirigé par un médecin-chef ; $2^{\circ}$ Service de chirurgie, dirigé par un chirurgien en chef; à ce service est rattaché une maternité où se font environ 200 accouchements par an.

Deux salles sont réservées pour hospitaliser les militaires de la place.

Un laboratoire installé pour effectuer les analyses chimiques et bactériólogiques est annexé à la pharmacie. Ce laboratoire est en même temps utiiisé comme laboratoire municipal d'hygiène ; il est dirigé par le pharmacien de l'hòpital.

C'est l'évolution et les améliorations successives apportées dans l'approvisionnement en lait de cet hôpital que nous voulons étudier ici.

Quand, en 1922, on jette un regard 20 ans en arriere sur les cahiers des charges qui servent de base à l'adjudication de la fourniture du lait aux hospices de Dieppe, on ne trouve, jusqu'en 1913, aucunes conditions spéciales sérieuses imposées aux adjudicataires pour servir un lait propre et non fraudé.

De 1902 à 1913, seule la mention suivante était indiquée au cahier des charges : a Le lait devra être pur et non écrémé, il devra donner au moins 9 degrés de erème au crémomètre de Chevallier en 24 heures.

"Dans le cas où le lait ne remplirait pas ces conditions, l'adjudicalaire serait passible d'une amende de 30 francs pour chaque contravention. ")

On sait combien le crémomètre a peu de valeur comme moyen de contròle; des causes nombreuses influent et modifient la montée de la crème, et il est difficile de se faire une opınion sur la pureté chimique d'un lait par le simple examen au crémomètre.

De plus, en exigeant un lait dosant simplement $9^{\circ}$ au crémomètre, l'hôpital laissait à son fournisseur une très large marge pour pratiquer une double fraude par mouillage et par écrémage.

En effet, le lait des vaches du pays de Caux et celles du pays de Bray titre $14^{\circ} 15$ et même $16^{\circ}$ au crémomètre; il était alors facile au fournisseur des Hospices d'écrémer son lait de 10 à $20 \%$ et de le mouiller à $10 \%$, tout en satisfaisant encore aux exigences du cahier des charges.

Quant aux qualités hygiéniques de fraicheur et de propreté que doit posséder un lait vraiment digne de ce nom, il n'en est nulle- 
ment fait mention, car l'administration ne soupçonnait pas qu'elle était en droit et qu'il était même de son devoir, d'exiger des conditions particulières sur ce point.

Aussi, jusqu'en 1913, le lait fourni aux Hospices pouvait être mouillé, écrémé, malpropre et pas trais, l'administration ne possédait aucun moyen de contrôle certain, ni aucune clause pour se défendre contre la malhonnêteté, la négligence et l'insouciance de son fournisseur.

Dans le cahier des charges de l'adjudication pour l'année 1913, l'administration a bien voulu, en plus des conditions générales imposées à toutes les adjudications, ajouter les conditions particulières suivantes :

\section{GONDITIONS PARTIGULIËRES}

Anticle 18. - Le lait livré à l'hôpital devra être le produit intégral de la traite des vaches bien portantes, bien nourries et non surmenées; il doit être recucilli proprement et ne doit pas contenir de colostrum.

Art. 19. - Il est interdit d'ajouter ou de retrancher au lait quelque substance que ce soit.

Art. 20. - Les vases destinés à contenir le lait doivent être emboutis et sans angles ; ils serunt étamés à l'étain fin ; il est interdit, pour leur bouchage, d'employer du linge ou autres objets, à l'exception du papier sulfurisé neuf. Le bouchage avec du caoutchouc contenant du plomb est aussi interdit.

Arı. 21. - Les vases destinés au transport du lait seront hermétiquement clos par des courercles métalliques fermant à pression ou à glissement. Ces vases serout tenus dans la plus parfaite propreté ; après chaque livraison, ils doivent être litvés à.l'intérieur el à l'extérieur, à l'eau bouillante.

Art. 22 - Les voitures qui transportent le lait doivent ètre tenues avec la plus grande propreté ; on ne devra transporter, avec le lait ou les vases vides, aucune marchandise capable de souiller le chargement.

Art. 23. - La Cominission administrative pourra, le cas échéant, faire visiter par une personne compétente, les animaux, les étables, la laiterie, les voitures de ladjudicataire.

Art. 24. - Le lait livré le matin sera exclusivement le produit de la traite du matin; le mélange de la traite du soir avec celle du matin ne sera pas accepté.

Art. 25. - Le lait livré l'ap ès-midi devra être le produit exclusif de la traite du midi.

Art. 26. - Le lait devra avoir la composition chimique suivante, au litre :

Lait du malin : Densilé : 1029 à 1033; beurre: 30 à 35 grammes, selon les saisons ; extrait sec : 130 grammes ; extrait dégraissé : 90 grammes; point eryoscopique : 0,54 à 0,58 .

Art. 27. - Au point de vue de ses qualités biologiques et hygiéniques, le 
lait devra répondre toute l'année aux réactions suivantes: $18^{\circ}$ Dornic; essai de Barthel: décoloration du bleu, en trois heures au moins; essai catalasimétrique : $2 \mathrm{~cm}^{3}$ d'oxygène pour $10 \mathrm{~cm}^{3}$ de lait.

C'est d'ailleurs à cette composition et à ces essais que doit répondre tout bon lait non fraudé, proprement recueilli et conservé avec soin.

Art 28. - Le lait est régulièrement contrôlé par le laboratoire municipal d'hygiène; s'il est reconnu mouillé ou écrémé, un prélèvement en quatro échantillons sera effeclué par l'adjoint chargé de la répressiou des fraudes, afin de permettre les poursuites judiciaires prèvues par la loi du 5 août 1905. Si le lait ne répond pas aux qualités hygiéniques et de frẩcheur indiquée plus haut, ladjudicataire sera passible d'une amende de trente francs ( $30 \mathrm{fr}$ ) par chaque contravention.

Monsieur le Docteur en Pharmacie Gurllaume,qui, dans son étude «Le lait à Rouen et en Seine-Inférieure », mentionne ces conditions, les fait suivre du commentaire suivant:

( Nous constatons, et cela avec une réelle satisfaction, que l'analyse chimique n'est plus employée seule pour assurer la réception du lait et que le contrôle hygiénique, avec ses trois essais: acidité, épreuves de la réductase et de la catalase, intervient également.

« Nous saisissons aussitôt la différence et le but de ces deux examens : l'analyse chimique ayant pour objet de réprimer la fraude, (écrémage, mouillage), alors que les essais biologiques doivent surtout punir les négligences coupables inconscientes le plus souvent) du fournisseur en le rendant passible d'une amende, et, en cas de récidive, en entraînant la résiliation du contrat, si son lait ne présente pas les qualités de fraîcheur voulue. »

C'est en se conformant à ces conditions, que l'adjudicataire a effectué ses livraisons aux hospices depuis 1913 jusqu'en 1919. Le contrôle du laboratoire permettait de s'assurer que les clauses du marché étaient bien remplies.

A partir du mois de septembre 1919, l'administration des hospices décida d'exploiter une ferme d'environ 40 hectares, que l'hôpital possède à Janval, aux portes de la ville, afin de produire elle-même le lait nécessaire à tout l'établissement.

Cette exploitation a été organisée pour produire un lait non fraudé, propre et frais, avec le contròle permanent du laboratoire.

Le cheptel, en 1919, se compose de 12 vaches, toutes tubereulinées et examinées par le vétérinaire sanilaire de la ville. 
Les saillies sont espacées pour qu'il y ait un vêlage à peu près chaque mois ; de cette façon, la quantité de lait est à peu près uniforme en toute saison. Les génisses sontélevées à la ferme, afin d'augmenter le cheptel économiquement et pour faire disparaitre les vieux sujets. Les vaches sont maintenues à l'herbage aussi longtemps que possible, elles ne rentrent à l'étable que lorsque l'herbe fait défaut. Le lait, pendant que les bêtes sont au pâturage, est de meilleure qualité et beaucoup plus propre, le pis des vaches étant peu souillé à l'herbage.

Pendant la stabulation, la nourriture se compose de paille, foin, betteraves, fourrages et d'un peu de tourteau de lin.

Les étables qui renferment 8 bêtes sont très éclairées; il y a 4 fenêtres par étable. Les auges, munis de cornadis, empêchant les bêtes gourmandes de voler la ration de leur voisine, sont surmontées d'une rigole où circule de l'eau courante (cette ferme possède en effet l'heureux privilège de disposer de l'eau potable de la ville).

Un lavabo, disposé dans l'étable, permet au vacher de se laver les mains avant la traite.

Les vachers ne couchent pas dans l'ètable, mais dans une chambre voisine, d'où ils peuvent surveiller ce qui se passe dans les vacheries.

La laiterie, installée sans luxe inutile, a été organisée pour conserver le lait dans de bonnes conditions.

Ellc possède un refrigérant, refroidi à l'aide de l'eau de Dieppe, dont la température ne dépasse jamais $11^{\circ}$.

Un bac en ciment, dans lequel circule un courant d'eau froide, occupe tout un còté de la laiterie.

Une écrémeuse centrifuge, une baratte, un malaxeur, une table en verre, permettent de faire du beurre quand il y a un excès de lait.

Enfin, à la porte de la laiterie, se trouve un appareil à rincer les brocs.

Toutes les vaches sont traites matin et soir, les nouvelles vélées sont également traites à midi.

Aussitôt la traite du soir ou du midi effectuée, soit à l'herbage, soit à l'étable, le lait est apporté à la laiterie, et, après filtration, il est envoyé sur le refrigérant. La température à la sortie du refrigérant est de 13 à $15^{\circ}$. Le lait est recueilli dans des brocs de 20 litres qui sont plongés dans le bac en ciment où circule l'eau à $11^{\circ}$. Les 
brocs y séjourneront jusqu'au lendemain matin. La traite du matin est effectuée vers 6 heures, le lait n'est pas refroidi, car il est rendu à l'hôpital à 7 heures, en même temps que le lait de la veille. Les

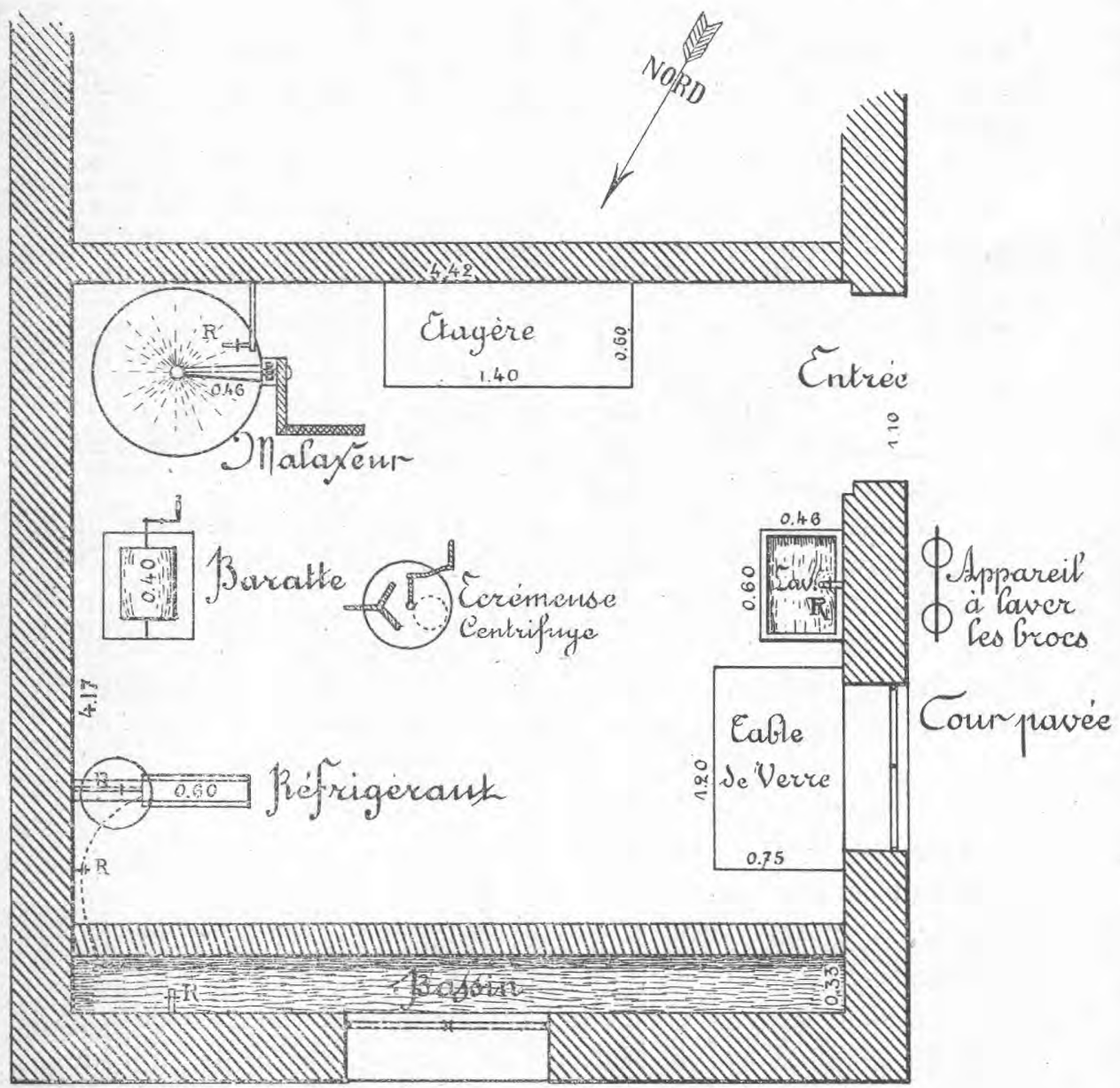

traites ne sont jamais mélangées, et les brocs portent des étiquettes pour chaque traite. La voiture qui transporte le lait est exclusivement réservée à cet usage ; la distance entre la ferme et l'hôpital n'est que de 2 kilomètres, le lait n'est donc nullement modifié pendant le transport.

Arrivé à l'hôpital vers $7 \mathrm{~h}$. 15, lẻ lait est divisé en deux lots : 
$1^{\circ}$ Une partie de la traite du matin est dirigée vers la pharmacie : c'est le lait dénommé lait pour enfants. Dans une salle spéciale, une sœur divise aussitôt le lait en autant de biberons qu'il est nécessaire pour chaque têtée, pour les différents enfants de la crèche et de la maternité. Les biberons sont stérilisés au bainmarie pendant 40 minutes, et refroidi à $11^{\circ}$ aussi vite que possible, grâce à un courant d'eau froide qui déplace l'eau bouillante.

Cette opération est terminé entre 8 h. $1 / 2$ et 9 heures, soit au plus, 3 heures après que le lait a été trait. Ce lait a donc une flore bactérienne insignifiante lorsque commence la stérilisation.

Dès l'ouverture des brocs, la sœur prélève chaque jour un échantillon du lait, qui est envoyé au laboratoire installé dans une pièce voisine.

2. Le reste du lait de la traite du matin, ainsi que celui de la traite du soir et du midi, sont dirigés vers la cuisine Placé dans des bassines, le lait subit une ébullition de 5 à 10 minutes; transvasé dans des brocs propres, il est aussitôt transporté à la sommellerie où il arrive environ à $80^{\circ}$, il est passé au refrigérant et refroidi vers $15^{\circ}$.

Mis en carafes d'un litre, il est distribué avant 9 heures du matin dans les différents services de l'hỏpital.

Ce lait est dénommé lait pour malades.

A son arrivée à la cuisine, un échantillon séparé des traites du matin, du midi et du soir, a été recueilli et envoyé au laboratoire.

Au laboratoire, le lait subit le contrôle suivant :

Il y a tous les jours 4 échantillons à surveiller :

$1^{\circ}$ Lait pour enfants ; $2^{\circ}$ Lait du matin ; $3^{\circ}$ Lait du midi ; $4^{\circ}$ Lait du soir.

Chaque échantillon est examiné :

$1^{\circ}$ Au point de vue de l'acidité par le procédé Dornic ;

$2^{\circ} \mathrm{Au}$ point de vue de l'épreuve de la réductase par le procédé de Barthel ;

$3^{\circ}$ Dans 4 bocaux renfermant du bi-chromate et correspondant chacun à une traite, on place environ $10 \mathrm{~cm}^{3}$ de lait des différents échantillons apportés au laboratoire, et toutes les 3 ou 4 semaines, le lait de ces bocaux est analysé au Gerber, au point de vue de sa teneur en matière grasse.

S'il y a des anomalies, on fait une analyse complète. Enfin, une 
ou deux fois par semaine, 1 litre du lait pour enfants est filtré dès sa réception sur le lacto-sédimentateur à ouate. Chaque rondelle de ouate est conservée et on peut de la sorte, à l'aide d'un témoin, voir si la traite est toujours faite proprement.

La quantité de lait produite est d'environ 35.000 litres par an, quantité suffisante pour les besoins de l'hòpital. D'ailleurs, quand le cheptel donnera son plein rendement, la production du lait sera plus importante, puisque la ferme peut nourrir 20 vaches adultes fournissant chacune de 2.500 à 3.000 litres de lait par an.

Grâce au contrôle permanent du laboratoire, depuis 2 ans l'acidité du lait s'est toujours maintenu dans les environs de $20^{\circ}$ Dornic.

Pendant les journées les plus chaudes, le lait du soir décolorait parfois le bleu de méthylène après 2 heures; pendant les saisons froides, la décoloration ne se faisait qu'après 10 à 12 heures.

Le lait livré aux malades et surtout celui destiné aux enfants, répond donc aux qualités de fraicheur et de pureté microbienne que l'on doit exiger d'un lait destiné à la puériculture.

La moyenne de toutes les analyses chimiques a fourni les chiffres suivants :

Matin : 37 grammes de beurre par litre.
Midi : 46 -
Soir: 42 -

Soit une teneur de 41 grammes par litre pour le lait des 3 traites. Quand l'hôpital achetait son lait, la teneur en beurre dépassait rarement 31 grammes par litre, car le lait provenait toujours, ou soit disant toujours, de la traite du matin.

C'est donc une différence en plus de 10 grammes de beurre par litre, dont bénéficient les malades de l'hòpital.

Si on ramenait le lait de la ferme à la même teneur en beurre que celle du lait, d'avant 1919, l'hôpital aurait produit, grâce à cet écrémage partiel, 350 kilogrammes de beurre, représentant une valeur marchande d'au moins 5.000 francs. Ces chiffres sont suffisamment éloquents pour montrer que la collaboration du laboratoire et du laitier producteur donnent des résultats autrement probants que le contrôle par le crémomètre de Chevallier et ses $9^{\circ}$ de crême. 